diffusing carbonium ions by acetonitrile solvent followed by hydrolysis (the Ritter reaction starting from an unactivated $\mathrm{C}-\mathrm{H}$ bond). The other minor byproducts including those derived from photooxidation of the tetra- $n$-butylammonium cations and the acetonitrile solvent as well as a small percentage of the epimerization product arise from freely diffusing intermediate radicals.

Not shown in Scheme III are the processes involving reoxidation of the reduced [or photoreduced] polyoxotungstates. Reoxidation can be facilitated by (1) reaction with $\mathrm{O}_{2}$ and protons, evolved from the dehydrogenation reactions, to form $\mathrm{H}_{2} \mathrm{O}$, (2) reaction solely with protons to form $\mathrm{H}_{2}$, or (3) in some circumstances, by reaction with other oxidants. These processes, although peripheral to the novel chemistry in the complex redox processes which we have elaborated in this paper, are nevertheless significant for they limit the overall rate of catalytic turnover.
The substantial degree to which the first and second redox potentials, protonation states, and other characteristics of the polyoxometalates that affect the relative rates of the key processes in Scheme III and analogous processes in other reactions can be systematically altered through rational syntheses of polyoxometalates suggests potential applications of these complex inorganic molecules to transformations of organic materials in synthesis and various technologies.

Acknowledgment. We thank the National Science Foundation (Grant CHE-9022317) for support.

Registry No. cis-1, 1579-21-1; trans-1, 16021-08-2; 2, 13837-12-2; 3, 1196-55-0; DTBO, 1876-22-8; $\mathrm{Q}_{3} \mathrm{PW}_{12} \mathrm{O}_{40}, 53749-37-4 ;\left(\mathrm{NH}_{4}\right)_{5} \mathrm{P}_{2} \mathrm{~W}_{18-}$ $\mathrm{O}_{62}, 110294-54-7 ; \mathrm{Q}_{4} \mathrm{~W}_{10} \mathrm{O}_{32}, 68109-03-5 ; \mathrm{Na}_{4} \mathrm{~W}_{10} \mathrm{O}_{32}, 126752-51-0 ; \mathrm{D}_{2}$, 7782-39-0; cis-decalin, 493-01-6; trans-decalin, 493-02-7.

\title{
Influence of Geometry on the Emitting Properties of 2,3-Naphthalimides
}

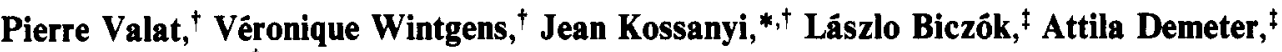 \\ and Tibör Bérces ${ }^{\ddagger}$
}

Contribution from the Laboratoire de Photochimie Solaire, E.R. 241, CNRS, 2-8 rue Henry

Dunant, 94320 Thiais, France, and Central Research Institute for Chemistry, Hungarian Academy of Sciences, Pusztaszeri ut 59-67, 1025 Budapest, Hungary.

Received November 20, 1990. Revised Manuscript Received September 23, 1991

\begin{abstract}
The luminescence properties of 2,3-naphthalimides have been studied using picosecond and nanosecond spectroscopies. In acetonitrile solution $N$-phenyl-2,3-naphthalimide (3) is found to emit dual fluorescence with emission maxima at 385 and $490 \mathrm{~nm}$, respectively. The short-wavelength emission corresponds to the known fluorescence of the naphthalimides and is demonstrated for 3 to originate from a molecular conformation in which the phenyl substituent and the naphthalimide skeleton are orthogonal to each other. The long-wavelength emission is assumed to originate from a singlet excited state formed by a ca. $90^{\circ}$ rotation of the phenyl group so that the two moieties are coplanar. Only a small dipole moment change is found between this excited state and the ground state. Only short-wavelength emission is observed with a lifetime in the nanosecond range as in the case of 1 and 2 when phenyl rotation is blocked with a bulky ortho tert-butyl group (compound 4). Increasing the viscosity of a glycerol/ethanol medium enhances both the efficiency and the lifetime of the short-wavelength emission of 3. It appears that at $77 \mathrm{~K}$ the emission originates directly from the Franck-Condon state. At room temperature, the other two emitting species are shown to arise from the Franck-Condon state by competitive intramolecular geometrical relaxation processes. Structures 5 and 6 are tentatively put forward to explain the formation of naphthazepinedione 8 by a $2 \pi+2 \pi$ photochemical cycloaddition reaction.
\end{abstract}

\section{Introduction}

The photochemistry of imides has received much attention during the last two decades, ${ }^{1-4}$ especially their inter- and intramolecular photoreduction reactions ${ }^{5-10}$ and their photoadditions to alkenes which leads to oxetanes, thus illustrating the reactivity of their carbonyl $n, \pi^{*}$ state. In addition, phthalimides and naphthalimides show typical photoreactivity with olefins leading to dihydrobenzazepinedione-type molecules ${ }^{11-17}$ following insertion of the alkene into the N-C bond of the imide function. Photoreductions, involving the abstraction of an allylic hydrogen atom of the olefin followed by the coupling of the resulting two radicals, have been shown ${ }^{17}$ to depend upon the redox potential of the alkene and to arise from photoinduced electron transfer. On the other hand, benzazepinedione formation has been postulated to occur through a $2 \sigma+2 \pi$ concerted cycloaddition. ${ }^{13,14}$ Extension of the $\pi$ conjugation using naphthalimides has shown a fundamental difference in reactivity depending on the position of the imide moiety in the molecule. ${ }^{5,11,17}$ Thus, irradiation of 2,3naphthalimides in the presence of olefins in methanol solution

\footnotetext{
${ }^{\dagger}$ CNRS.
${ }^{t}$ Hungarian Academy of Sciences.
}

yields oxetanes, naphthazepinedione, and solvent-trapped adducts, ${ }^{5,11}$ while the 1,8-naphthalimides give only tetracyclic imides

(1) Coyle, J. D. Pure Appl. Chem. 1988, 60, 941.

(2) Coyle, J. D. In Synthetic Organic Photochemistry; Horspool, W. M., Ed.; Plenum: New York, 1983; p 259.

(3) Mazzocchi, P. H. In Organic Photochemistry; Padwa, A., Ed.; Marcel Dekker: New York, 1981; Vol. 5, p 421.

(4) Kanaoka, Y. Acc. Chem. Res. 1978, 11, 407

(5) Machida, M.; Nakamura, M.; Oda, K.; Takeshi, H.; Ohno, K.; Nakai, H.: Sato, Y. Kanaoka, Y. Heterocycles 1987, 26, 2683.

(6) Machida M.: Oda, K.: Kanaoka, Y. Tetrahedron 1985, $41,4995$.

(7) Mazzocchi, P. H.; Klingler, L. J. J. Am. Chem. Soc. 1984, 106, 7567.

(8) Hayashi, H.; Nagajura, S.; Kubo, Y.; Maruyrma, K. Chem. Phys. Lett. $1980,72,291$

(9) Kanaoka, Y.; Hatanaka, Y. J. Org. Chem. 1976, 41, 400.

(10) Kanaoka, Y.; Migita, Y.; Koyama, K. Tetrahedron Lett. 1973, 1193.

(11) Kubo, Y.; Suto, M.; Araki, T.; Mazzocchi, P. H.; Klingler, L.; Shook, D.; Edwards, M.; Somich, C. J. Org. Chem. 1986, $51,4404$.

(12) Maruyama, K.; Kubo, Y. J. Org. Chem. 1985, 50, 1426.

(13) Mazzocchi, P. H.; Klingler, L.; Edwards, M.; Wilson, P.; Shook, D. Tetrahedron Lett. 1983, 24, 143 .

(14) Mazzocchi, P. H.; Wilson, P.; Khachik, F.; Klinger, L.; Minamikawa, S. J. Org. Chem. 1983, 48, 2981 .

(15) Mazzocchi, P. H.; Minamikawa, S.; Wilson, P.; Bowen, M.; Narian, N. J. Org. Chem. 1981, 46,4846 


$$
10^{3} \varepsilon\left(\mathrm{M}^{-1} \mathrm{~cm}^{-1}\right)
$$

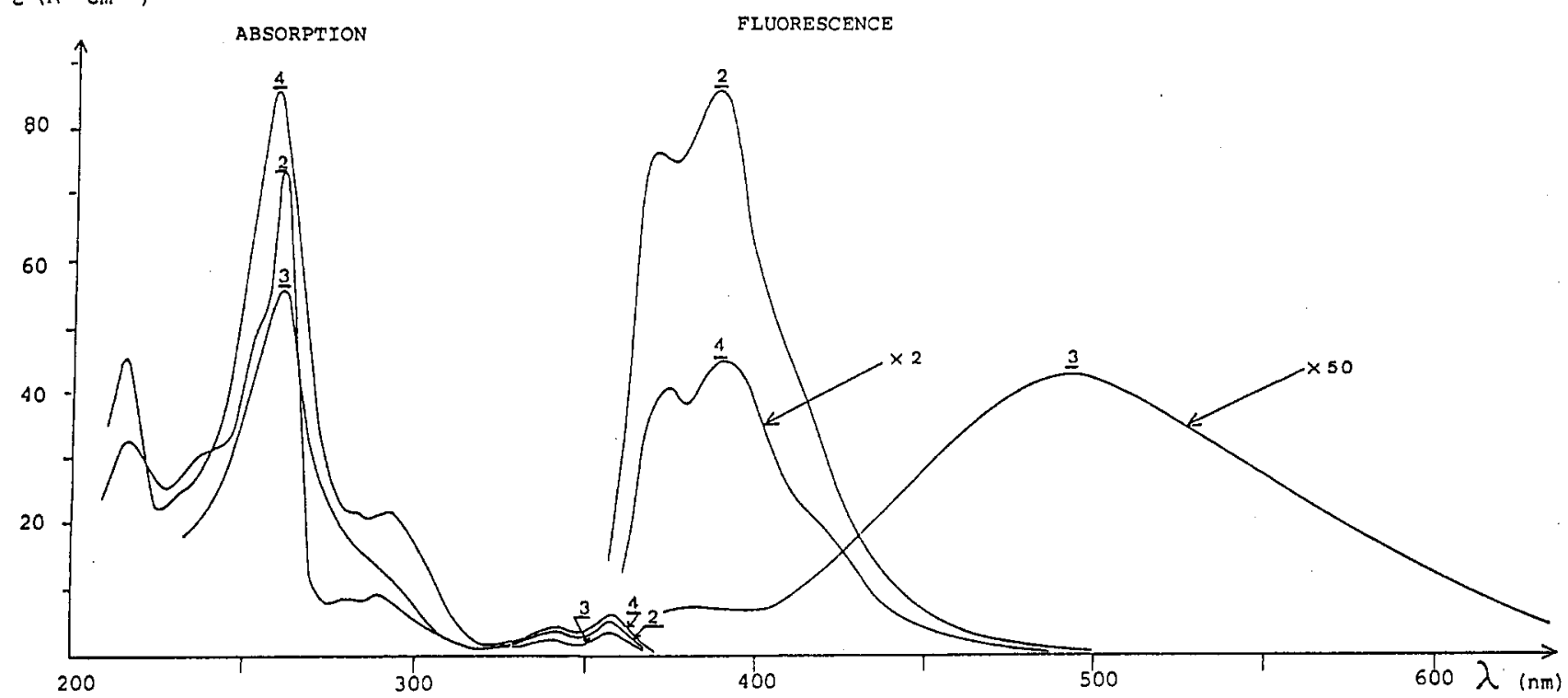

Figure 1. Absorption and fluorescence spectra of 2-4 at room temperature in acetonitrile solution.

by 1,4-addition of the olefin to the naphthalene ring together with methanol incorporation, ${ }^{17}$ and no naphthazepinedione was found in this case.

Phthalimides substituted on the nitrogen atom by a (dialkylamino)alkyl group probably react from a $\mathrm{n}, \pi^{*}$ singlet excited state since flash photolysis experiments indicate two transients - a triplet species and an isomeric imide resulting from hydrogen 1,3shift-which are unrelated to the intramolecular photochemical cyclization. $^{18}$

Surprisingly, little is known about the spectroscopic properties of the dicarboximides. The fluorescence emission of $N$-alkylphthalimides $^{19}$ has been reported recently. $N$-Phenyl-2,3naphthalimide shows fluorescence properties (vide infra) quite different from those of the corresponding $N-\mathrm{H}$ and $N$-methyl derivatives. For instance, 1 and 2 have a normal fluorescence emission around $385 \mathrm{~nm}$; however, the $N$-phenyl-substituted compound 3 presents a dual emission which is viscosity and temperature dependent. It is the aim of the present paper to rationalize this difference and to offer a plausible explanation in terms of molecular motions.

$$
\begin{aligned}
& \underline{1}=H \\
& \underline{2} R=C_{3} \\
& \underline{3} R=C_{6} H_{5} \\
& \underline{4} R=2,5-\text { di } R=\text { t.Bu-C }{ }_{6} H_{4}
\end{aligned}
$$

\section{Experimental Part}

The UV-visible absorption spectra were obtained using a Varian-Cary Model 219 apparatus. Fluorescence spectra were recorded with a Perkin-Elmer Model MPF 44B apparatus equipped with a DCSU-2 spectral

(16) Mazzocchi, H. P.; Bowen, M.; Narian, N. J. Am. Chem. Soc. 1977, 88,7063 .

(17) Somich, C.; Mazzocchi, P. H.; Ammon, H. L. J. Org. Chem. 1987, 52,3614 .

(18) Coyle, J. D.; Harriman, A.; Newport, G. L. J. Chem. Soc., Perkin Trans. 2 1979, 799.

(19) Berci Filho, P.; Toscano, V. G.; Politi, M. J. J. Photochem. Photobiol. A 1988, 43, 51 . correction unit and with a homemade photocounting spectral fluorimeter using the Princeton Applied Research 1140 A/B detection system. Fluorescence quantum yields were measured in ethanol and acetonitrile solutions by comparison with the emission from quinine sulfate (in $1 \mathrm{~N}$ aqueous sulfuric acid solution) for which a value 0.55 was taken. ${ }^{20}$

Phosphorescence spectra were obtained, at $77 \mathrm{~K}$ in a $95: 5$ butyronitrile/butyl acetate mixture, using a Perkin-Elmer MPF 44B apparatus equipped with a phosphorescence accessory which is coupled to an oscilloscope and permits phosphorescence decay measurements to be made at times as short as $3 \mathrm{~ms}$. Variable-temperature measurements were carried out with a liquid nitrogen cooled Dewar (Oxford Instrument) which enables measurements down to ca. $85 \mathrm{~K}$.

Singlet lifetimes were measured by excitation with a frequency-tripled pulsed YAG laser (from B. M. Industries) of 30-ps fwhm. For lifetimes greater than $1.5 \mathrm{~ns}$ the light was focused through a cylindrical lense onto the sample placed in front of a photodiode. The output from the photodiode was fed into a Tektronix 7912 AD digitized oscilloscope and the data, which were stored in an Apple II+ microcomputer, could be displayed on a Hewlett-Packard Model 7470A graphic plotter. Averaged decays could be analyzed directly by the microcomputer. For shorter lived fluorescent species, a TSN 506 streak camera was used with a ca. 10-ps time resolution, and the fluorescence was focused through an optical coupling on the slit of the streak camera and the data were transferred to a multichannel analyzer which enabled the accumulation of several decay measurements. In this way lifetimes of $50 \mathrm{ps}$ or more could be analyzed in the same way as described for data stored in the digitized oscilloscope.

Ethanol (Prolabo, RP grade), acetonitrile (Aldrich, HPLC grade), glycerol (Merck, fluorescence grade), and butyl chloride (Aldrich, HPLC grade) were used as received. Butyronitrile (Aldrich) and butyl acetate (Aldrich) were purified by chromatography over alumina and distilled before use.

Preparation of the Products. The preparation, purification, and characterization of compounds $1,21,2,22$ and $3^{23}$ have been already described. Flash chromatography over silica gel (elution by dichloromethane) and recrystallization from ethyl acetate were carried out as the last purification steps.

2-(2',5'-Di-tert -butylphenyl)-1H-benz[f]isoindole-1,3(2H)-dione (4). 2,3-Naphthalenedicarboxylic acid $(1.08 \mathrm{~g}, 0.005 \mathrm{M})$ was intimately mixed with 2,5-di-tert-butylaniline $(5.12 \mathrm{~g}, 0.025 \mathrm{M})$, and the resultant mixture was heated for $3 \mathrm{~h}$ at $180-200^{\circ} \mathrm{C}$. After cooling, the solid reaction mixture was ground up and added to $100 \mathrm{~mL}$ of aqueous $\mathrm{HCl}$ (1 N) and stirred at $60^{\circ} \mathrm{C}$ for $0.5 \mathrm{~h}$. The solid was then filtered and heated for $0.5 \mathrm{~h}$ at $50^{\circ} \mathrm{C}$ in $100 \mathrm{~mL}$ of aqueous $\mathrm{NaHCO}_{3}(1 \mathrm{M})$. After separation, the remaining solid $(1.26 \mathrm{~g}$, yield $65 \%)$ was purified by chromatography (elution with $\mathrm{CH}_{2} \mathrm{Cl}_{2}$ ) over silica gel, and the product

(20) Melhuish, W. H. J. Phys. Chem. 1961, 65, 229.

(21) Freund, M.; Fleischer, K. Annalen 1974, 402, 69

(22) Rigaudy, J.; Sparfeld, D. Tetrahedron 1978, 39, 2263.

(23) Cava, M. P.; Deana, A. A.; Muth, K. J. Am. Chem. Soc. 1959, 81 , 
Table I. Fluorescence (in Acetonitrile) and Phosphorescence (in 95/5 Butyronitrile/Butyl Acetate) Properties of Dicarboximides

\begin{tabular}{|c|c|c|c|c|c|}
\hline \multirow[b]{2}{*}{ compd } & \multicolumn{3}{|c|}{ fluorescence $\left(20^{\circ} \mathrm{C}\right)$} & \multicolumn{2}{|c|}{$\begin{array}{c}\text { phosphorescence } \\
(77 \mathrm{~K})\end{array}$} \\
\hline & $\lambda_{\max }(\mathrm{nm})$ & $\Phi_{\mathrm{f}}$ & $\tau_{\mathrm{f}}$ & $\lambda_{\max }(\mathrm{nm})$ & $\tau_{\mathrm{ph}}(\mathrm{s})$ \\
\hline 1 & 379 & 0.26 & $8.2 \mathrm{~ns}$ & $496,526,560$ & 3.2 \\
\hline 2 & 385 & 0.24 & $7.95 \mathrm{~ns}$ & $495,523,554$ & 3.8 \\
\hline \multirow[t]{2}{*}{3} & 385 & $\leq 2 \times 10^{-4}$ & $\leq 50 \mathrm{ps}$ & & \\
\hline & 480 & 0.005 & $1.3 \mathrm{~ns}$ & $496,522,560$ & 3.7 \\
\hline 4 & 390 & 0.066 & $1.22 \mathrm{~ns}$ & $490,520,560$ & 3.5 \\
\hline
\end{tabular}

obtained was recrystallized from ethyl acetate: $\mathrm{mp} 225-227^{\circ} \mathrm{C}$; UV $\left(\mathrm{CH}_{3} \mathrm{CN}\right), \lambda_{\max }\left(\epsilon, \mathrm{L} \cdot \mathrm{mol}^{-1} \cdot \mathrm{cm}^{-1}\right) 215 \mathrm{~nm}(45200), 258(85700), 340$ (3700), 356 (5700); IR $\left(\mathrm{CHCl}_{3}\right)$ 3000, 2940, 1750, 1690, 1595, 1585, $1490,1410,1395,1360,1345,1180,1140,1110,900,825 \mathrm{~cm}^{-1}$.

\section{Results}

The literature contains very little information on the spectroscopic properties of the naphthalimides. Therefore, by way of illustration, the absorption and fluorescence spectra of 2 methyl-1 $H$-benz [f] isoindole-1,3(2H)-dione (2, $N$-methyl-2,3naphthalimide) and its $N$-phenyl 3 and $N$-( $2^{\prime}, 5^{\prime}$-di-tert-butylphenyl) 4 derivatives in acetonitrile solution are given in Figure 1 .

A summary of the most important fluorescence and singlet-state properties of compounds 1-4 are given in Table I.

A. Absorption and Fluorescence. The absorption spectra of all the 2,3-naphthalimides, 1-4, in $\mathrm{CH}_{3} \mathrm{CN}$ or $\mathrm{CH}_{2} \mathrm{Cl}_{2}$ solution show a short-wavelength absorption maximum around $255 \mathrm{~nm}$ and a structured one at longer wavelength with maxima at 340 and $355 \mathrm{~nm}$. The fluorescence spectra of unsubstituted 2,3naphthalimide 1 and of its $N$-methyl derivative 2 are mirror images of their long-wavelength absorption spectra. The fluorescence decays with a lifetime of ca. $8 \mathrm{~ns}$.

$N$-Phenyl-2,3-naphthalimide (3) shows a dual fluorescence (Figure 1). At room temperature, a very weak fluorescence $\left(\Phi_{\mathrm{f}}\right.$ $<2 \times 10^{-4}$ ) is observed at $350-410 \mathrm{~nm}$, in addition to a much stronger broad and structureless fluorescence band situated in the 420-620-nm range, with a maximum at $490 \mathrm{~nm}$.

In both $\mathrm{CH}_{2} \mathrm{Cl}_{2}$ and $\mathrm{CH}_{3} \mathrm{CN}$ solution, the weak emission band observed for 3 at room temperature between 350 and $400 \mathrm{~nm}$ is analogous to that of $\mathbf{1}$ and $\mathbf{2}$, but a significant difference is observed in their lifetime: The short-wavelength emission of $\mathbf{3}$ has a lifetime shorter than 50 ps (technological limit) while the long-wavelength emission decays with a lifetime of $1.3 \mathrm{~ns}$ in acetonitrile and 2.1 $\mathrm{ns}$ in dichloromethane solution. By blocking the rotation of the $N$-phenyl group with a 2'-tert-butyl substituent (compound 4), the long-wavelength emission is suppressed and the lifetime of the short-wavelength fluorescence is now on a nanosecond time scale.

Solvent Effect. The shift of the long-wavelength fluorescence band of $\mathbf{3}$ as a function of solvent polarity has been examined by using many solvents varying from nonpolar ones (hexane, methylcyclohexane) to strongly polar ones (acetonitrile, propylene carbonate). The position of the fluorescence maximum $\bar{\nu}_{\max }$ has been plotted versus Lippert's $\Delta f$ function $^{24}$

$$
\bar{\nu}_{\max }=\frac{2\left(\mu_{\mathrm{e}}-\mu_{\mathrm{g}}\right)^{2}}{h c a^{3}}\left[\frac{\epsilon-1}{2 \epsilon+1}-\frac{n^{2}-1}{2 n^{2}+1}\right]=\frac{2\left(\mu_{\mathrm{e}}-\mu_{\mathrm{g}}\right)^{2}}{h c a^{3}} \Delta f
$$

where $\mu_{\mathrm{g}}$ and $\mu_{\mathrm{e}}$ refer to the dipole moments of the ground and excited states of the molecule, respectively, $h$ is Planck's constant, $c$ is the velocity of light, $a$ is the radius of Onsager cavity which fits the molecule, and $\epsilon$ and $n$ are the solvent dielectric constant and refractive index, respectively. As can be seen from Figure 2 , a reasonably linear plot is obtained. The variation in $\bar{\nu}_{\max }$ between hexane $(\epsilon=1.88)$ and propylene carbonate $(\epsilon=65.1)$ is rather small, ca. $900 \mathrm{~cm}^{-1}$. This corresponds to a change in dipole moment between the ground state and the singlet excited state of $3.4 \mathrm{D}$.

(24) Lippert, E. Z. Electrochem. 1957, 61, 962.

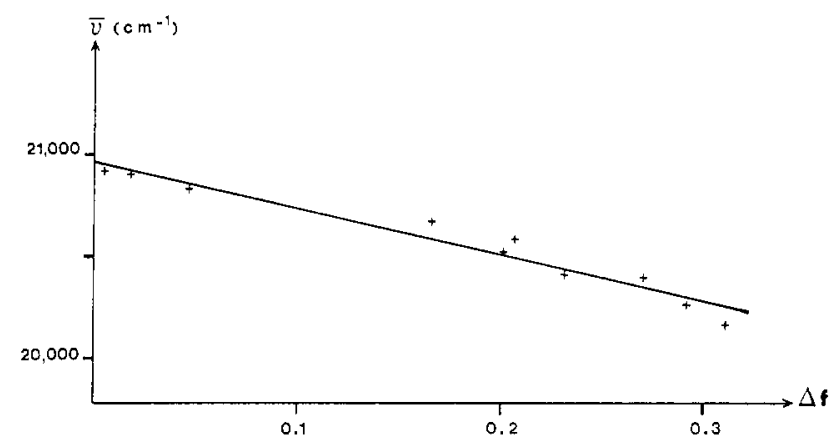

Figure 2. Plot of the position of the long-wavelength fluorescence maximum of 3 versus Lippert's $\Delta f$ expression.

B. Viscosity and Temperature Effects on the Fluorescence. The following ethanol/glycerol (v/v) mixtures were used: $100 / 0$, $50 / 50,33 / 67$, and $20 / 80$ to change the viscosity of the solutions at room temperature. As the viscosity of the solution increases, the intensity of the long-wavelength emission around $490 \mathrm{~nm}$ decreases and that of the short-wavelength emission around 405 $\mathrm{nm}$ increases (Figure 3). Simultaneously, the lifetime of the short-wavelength fluorescence increases from less than $50 \mathrm{ps}$ to $125 \mathrm{ps}, 180 \mathrm{ps}$, and $450 \mathrm{ps}$, respectively. However, no change in lifetime is found for the long-wavelength emission which remains at $1.3 \mathrm{~ns}$ in all four solutions.

The time-resolved study of the two fluorescences in the 50/50 $(\mathrm{v} / \mathrm{v})$ ethanol/glycerol mixture demonstrates that both emissions have rise times within the $50 \mathrm{ps}$ of the laser pulse (Figure 4).

In ethanol solution at room temperature, 3 emits mainly around $490 \mathrm{~nm}$ while, at $100 \mathrm{~K}$ or lower, the short-wavelength emission, analogous to that of 1 and 2, is mainly observed (Figure 5). Also, the lifetime of the short-wavelength emission increases from less than 50 ps at room temperature to several nanoseconds at $77 \mathrm{~K}$ while the long-wavelength fluorescence lifetime does not vary much when the temperature decreases from 293 to $135 \mathrm{~K}$.

C. Phosphorescence. All measurements were carried out in a $95 / 5$ butyronitrile/butyl acetate mixture. At $77 \mathrm{~K}$ in this mixture all compounds have a structured phosphorescence with three maxima around 492,524 , and $560 \mathrm{~nm}$ and a lifetime between 3.2 and $3.8 \mathrm{~s}$. The spectra are similar to that of naphthalene but red-shifted by ca. $25 \mathrm{~nm}$.

\section{Discussion}

The long-wavelength fluorescence could be attributed to a "twisted intramolecular charge-transfer" (TICT) state emission as found, for instance, in the case of the somewhat structurally related benzanilides. ${ }^{25}$ A TICT state contains two separated charged moieties within a molecule geometrically disposed to assure a minimum overlap of their orbitals. The emission from such a polar state occurs at long wavelengths and is strongly dependent on the solvent polarity. ${ }^{26}$ The small variation in $\Delta \mu$ (3.4 D) between the ground state and the excited state emitting at long wavelengths, as deduced from the plot of $\bar{\nu}_{\max }$ versus $\Delta f$, is incompatible with an emission originating from a TICT-type species since one would expect a rather large change in dipole moment ${ }^{26,27}$ for the molecule when one electric charge has been transferred from the donor moiety to the acceptor part of the molecule. Such a change in dipole moment would also cause a large shift $\left(3000-5000 \mathrm{~cm}^{-1}\right)$ in the position of the fluorescence maximum, i.e., larger than the $900 \mathrm{~cm}^{-1}$ observed for 3 when hexane is replaced by acetonitrile. For this reason, and others which are discussed below, a TICT state cannot be put forward to explain the dual emission observed here for 3 .

The appearance of the long-wavelength fluorescence can be attributed to the presence of the phenyl rotor in the molecule, and

(25) (a) Heldt, J.; Gormin, D.; Kasha, M. Chem. Phys. 1989, 136, 321; (b) J.Am. Chem. Soc. 1988, 110,8256.

(26) Rettig, W. Angew. Chem., Int. Ed. Engl. 1986, 25, 4971.

(27) Rettig, W. J. Mol. Struct. 1982, 84, 303. 


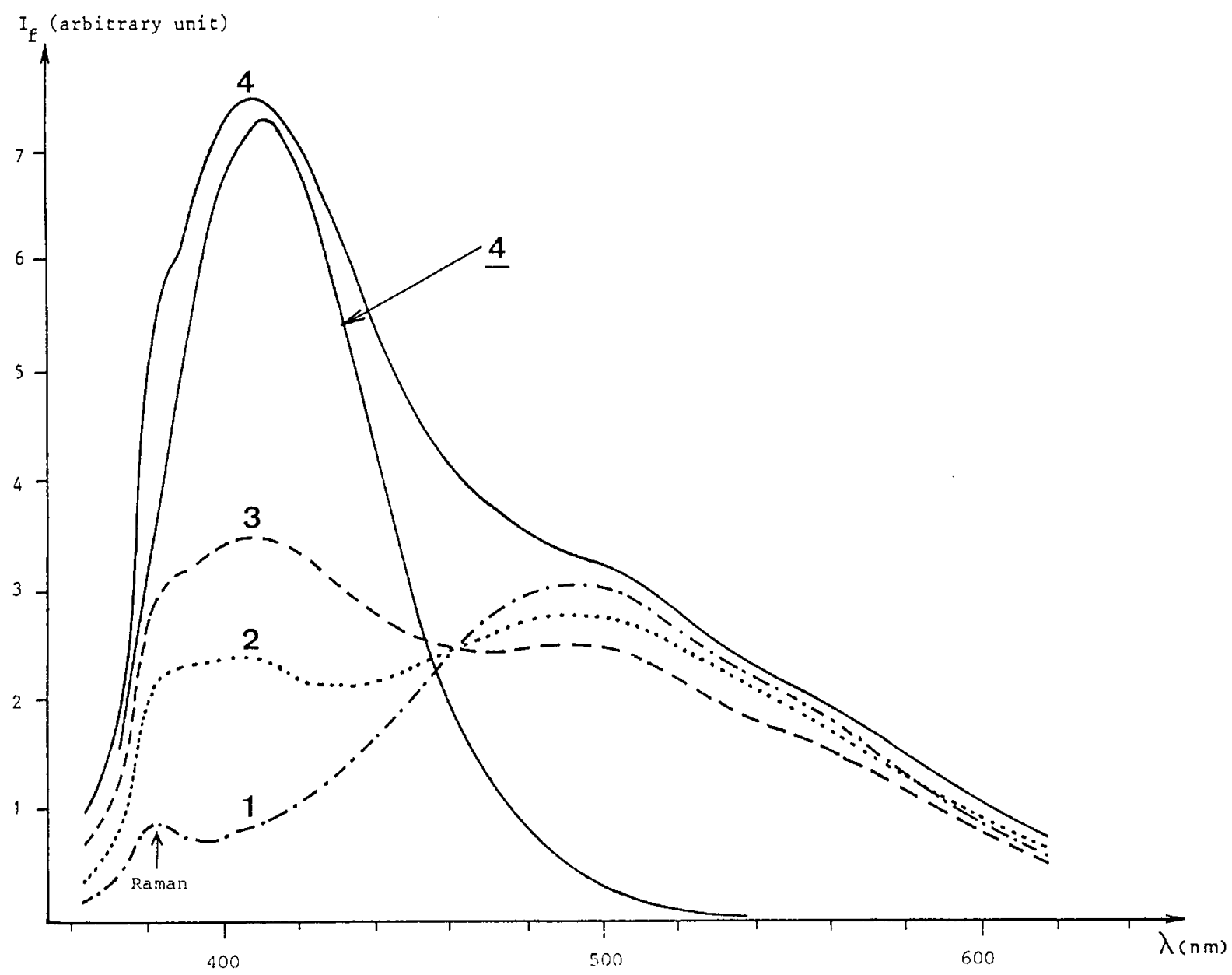

Figure 3. Fluorescence spectra of 3 in various glycerol/ethanol (v/v) mixtures: (1) $0 / 100$; (2) 50/50; (3) 67/33; (4) 80/20. For comparison, the spectrum of 4 in the $80 / 20$ mixture is given.
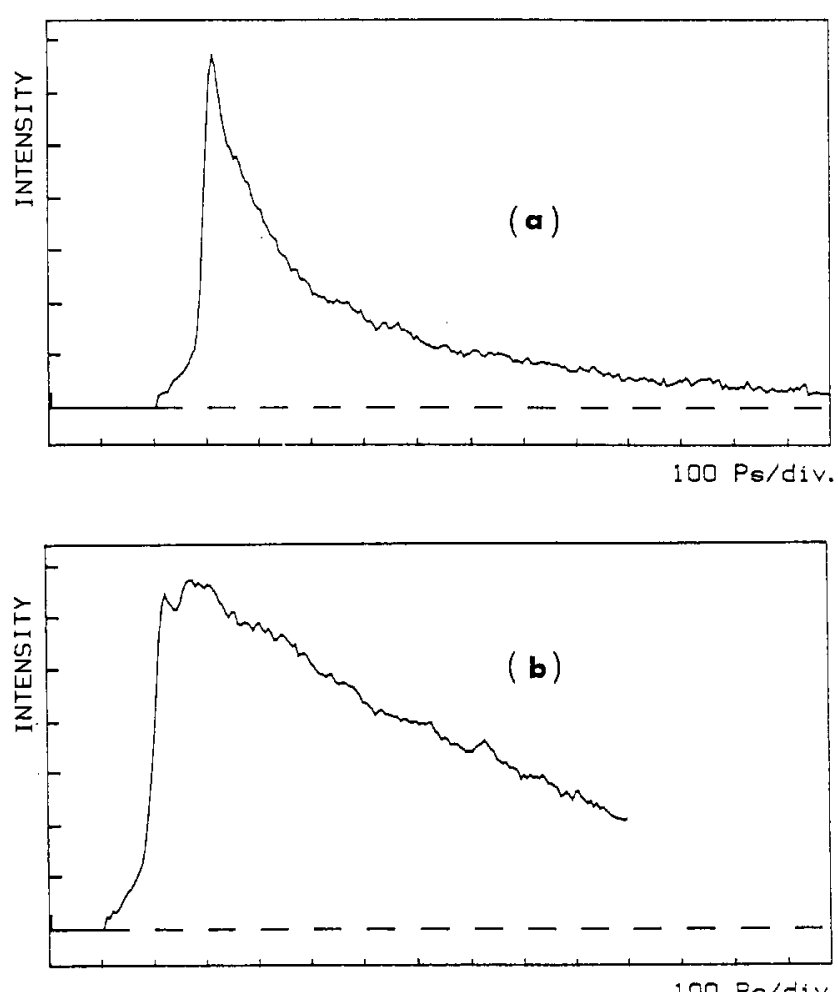

Figure 4. Growth and decay of the fluorescence of 3 in the 50/50 (v/v) glycerol/ethanol mixture at room temperature. Analysis: (a) in the $385-450-\mathrm{nm}$ range; (b) at $\lambda>475 \mathrm{~nm}$. a more appropriate model has to be found for explaining the dual emission.

Inhibition of the $\boldsymbol{N}$-Phenyl Rotation in 3. Several approaches have been used to demonstrate the importance of the rotation of the $N$-phenyl substituent of compound 3. One of them has been the study of $N$-(2',5'-di-tert-butylphenyl)-2,3-naphthalimide (4) in which the bulky $2^{\prime}$-tert-butyl group prevents the rotation of the $N$-phenyl substituent around the free $\mathrm{N}-\mathrm{C}$ single bond. In acetonitrile solution this compound exhibits only the short-wavelength emission, exactly at the fluorescence wavelengths of compounds 1 and 2. Its fluorescence quantum yield (0.066) and singlet lifetime $(1.2 \mathrm{~ns})$ in acetonitrile are at least 25 times higher than those of compound 3 which contains the $N$-phenyl which is free to rotate. In compound $4, \Phi_{\mathrm{f}}$ and $\tau_{\mathrm{s}}$ are still 4-5 times smaller than the corresponding values for 1 and 2. Thus, at this stage, the short-wavelength emission can be attributed to a molecular structure in which the 2,3-naphthalimide skeleton is not perturbed by the substituents on the nitrogen atom. In the case of compound 3 this implies that the short-wavelength emission originates from a structure in which no interaction exists between the two moieties of the molecule. This occurs when the $N$-phenyl substituent is in a plane orthogonal to the naphthalimide moiety. In such a case, if a TICT was developing with this appropriate structure, we should expect (i) the presence of a long-wavelength emission for compound 4 and (ii) an increase of this emission for compound 3 when the temperature is lowered to $77 \mathrm{~K}$. Since this is definitely not the case, one cannot attribute the long-wavelength fluorescence to a TICT state.

One possible hypothesis explaining the dual emission of 3 is that the Franck-Condon state formed by light absorption emits at short wavelengths (SW) and geometrical changes of this excited molecule could lead to a new species emitting at long wavelength 


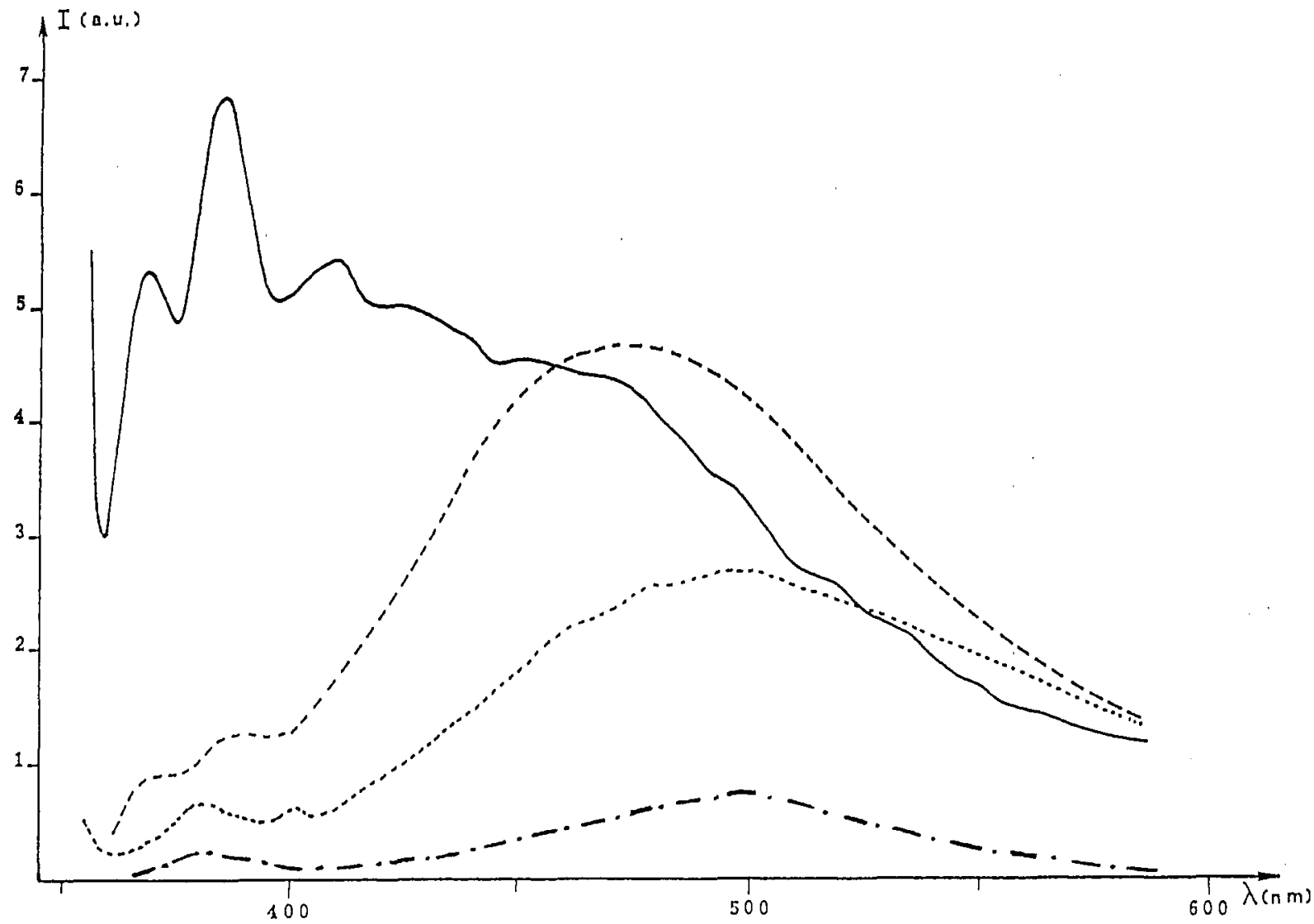

Figure 5. Fluorescence of compound 3 in ethanol solution at (-) $100 \mathrm{~K},(-.) .135 \mathrm{~K},(--) 220 \mathrm{~K}$, and (-.-) $293 \mathrm{~K}$.

(LW). If this was the case, then the time required by $\mathrm{SW}^{*}$ to decay would have been the same as the time needed for $\mathrm{LW}^{*}$ to grow in. Time-resolved experiments carried out on the two emissions of 3 in a viscous medium (1/1 ethanol/glycerol (v/v) mixture) indicate that both $\mathrm{SW}^{*}$ and $\mathrm{LW}^{*}$ are formed within the duration of the laser pulse ( $<50 \mathrm{ps}$ ) and decay in $125 \mathrm{ps}$ and 1.3 ns, respectively (Figure 4). This eliminates a direct path between the two emitting species. The same conclusion can be drawn if an intermediate between the two luminophors was involved: Thus, if we suggest that the sequence is $\mathrm{SW}^{*} \rightarrow \mathrm{X}^{*} \rightarrow \mathrm{LW}^{*}$ and that the intermediate $X^{*}$ is shorter lived than $S^{*}$, then the growth of $\mathrm{LW}^{*}$ would still correspond to the decay of $\mathrm{SW}^{*}$. On the other hand, if we consider the opposite situation, where $\mathrm{X}^{*}$ is somewhat longer lived than $\mathrm{SW}^{*}$, then the growing in of $\mathrm{LW}^{*}$ would be more easy to observe. Since both $\mathrm{SW}^{*}$ and $\mathrm{LW}^{*}$ have rise times within the 50-ps laser pulse, $\mathrm{SW}^{*}$ cannot be the precursor of $\mathrm{LW}^{*}$. These conclusions also eliminate SW* $^{*}$ as being the Franck-Condon state $A^{*}$. Therefore, the latter must generate two independent states, $\mathrm{SW}^{*}$ and $\mathrm{LW}^{*}$.

We therefore propose the following model: Light absorption promotes molecule $\mathrm{A}^{0}$ to its lowest singlet excited state which undergoes fast transition to the relaxed Franck-Condon state $A^{*}$. Then, in addition to the usual nonradiative deactivation processes, we assume that $A^{*}$ undergoes intramolecular rearrangements to two, geometrically different excited states $\mathrm{SW}^{*}$ and $\mathrm{LW}^{*}$, the second one resulting from the rotation of the phenyl group around the $\mathrm{N}-\mathrm{C}$ single bond. A similar process has been suggested to occur after excitation of phenyl-substituted biphenyls. ${ }^{28}$ However, contrary to the biphenyl case, it is the newly formed LW* state which emits the long-wavelength fluorescence after vibrational relaxation. That the long-wavelength fluorescence emission involves the rotation of the $N$-phenyl group is demonstrated by $N$-(2',5'-di-tert-butylphenyl)-2,3-naphthalimide (4) in which the $2^{\prime}$-tert-butyl substituent inhibits the rotation around the $\mathrm{N}-\mathrm{C}$ single bond. In acetonitrile solution, no emission originating from $\mathrm{LW}^{*}$

(28) Cooke, B. J.; Palmer, T. F. J. Photochem. 1984, 26, 149. is observed for this compound but only the SW* fluorescence at $390 \mathrm{~nm}$ with a quantum yield of 0.066 and a lifetime of $1.2 \mathrm{~ns}$.

Kinetic Scheme. The kinetic scheme which explains the dual fluorescence would be

$$
\begin{gathered}
\mathrm{A}^{0}+h \nu \rightarrow \mathrm{A}^{*} \\
\mathrm{~A}^{*} \stackrel{k}{\longrightarrow} \mathrm{A}^{0} \\
\mathrm{~A}^{*} \stackrel{k^{\prime}}{\longrightarrow} \mathrm{SW}^{*} \\
\mathrm{SW}^{*} \stackrel{k_{f}^{\prime}}{\longrightarrow} \mathrm{A}^{0}+h \nu^{\prime} \\
\mathrm{SW}^{*} \stackrel{k_{\mathrm{m}}^{\prime}}{\longrightarrow} \mathrm{A}^{0} \\
\mathrm{~A}^{*} \stackrel{k^{\prime \prime}}{\longrightarrow} \mathrm{LW} \\
\mathrm{LW}^{*} \stackrel{k_{f}^{\prime \prime}}{\longrightarrow} \mathrm{A}^{0}+h \nu^{\prime \prime} \\
\mathrm{LW}^{*} \stackrel{k_{\mathrm{mit}}^{\prime \prime}}{\longrightarrow}\left(\mathrm{A}^{0}\right)
\end{gathered}
$$

On the basis of this scheme the short-wavelength and the longwavelength fluorescence quantum yields, $\Phi_{\mathrm{f}}{ }^{\prime}$ and $\Phi_{\mathrm{f}}{ }^{\prime \prime}$, respectively, are

$$
\Phi_{\mathrm{f}}^{\prime}=\Phi_{\mathrm{SW}}{ }^{\prime} k_{\mathrm{f}}^{\prime} \tau^{\prime}
$$

and

$$
\Phi_{\mathrm{f}}{ }^{\prime \prime}=\Phi_{\mathrm{LW}}{ }^{\prime \prime} k_{\mathrm{f}}{ }^{\prime \prime} \tau^{\prime \prime}
$$

where $\tau$ and $\tau^{\prime \prime}$ are the lifetimes of $S W^{*}$ and $L W^{*}$ and $\Phi_{S W^{\prime}}$ and $\Phi_{L W^{\prime \prime}}$ designate the quantum yields for the formation of the SW* and $\mathrm{LW}^{*}$ states, respectively:

$$
\begin{aligned}
\Phi_{S W^{\prime}} & =\frac{k^{\prime}}{k+k^{\prime}+k^{\prime \prime}} \\
\Phi_{L W^{\prime \prime}} & =\frac{k^{\prime \prime}}{k+k^{\prime}+k^{\prime \prime}}
\end{aligned}
$$


The ratio of the two fluorescence yields can be expressed as

$$
\frac{\Phi_{\mathrm{f}}^{\prime \prime}}{\Phi_{\mathrm{f}}^{\prime}}=\frac{k^{\prime \prime}}{k^{\prime}} \frac{k_{\mathrm{f}}^{\prime \prime}}{k_{\mathrm{f}}^{\prime}} \frac{\tau^{\prime \prime}}{\tau^{\prime}}
$$

Thus, if the Franck-Condon state decays to only $L^{*}$ and $\mathrm{SW}^{*}$, any change which hinders the phenyl rotation should decrease the efficiency of reaction 6 and the quantum yields of all the processes originating from $L W^{*}$ including $\Phi_{f}^{\prime \prime}$. Therefore, the efficiency of the short-wavelength emission would increase.

The origin of the triplet state is a little puzzling, since it could be generated from $\mathbf{A}^{*}, \mathrm{SW}^{*}$, or $\mathrm{LW}^{*}$ or even from all three states. The phosphorescence observed at low temperature $(<100 \mathrm{~K})$ must originate from $\mathrm{A}^{*}$, since the other two, $\mathrm{LW}^{*}$ or $\mathrm{SW}^{*}$, are no more populated at this temperature (vide infra).

The decay time of the SW* state of 3 is at least 2 orders of magnitude shorter than the singlet lifetimes of the unsubstituted 1 and the $N$-methyl derivative 2 . In terms of the reaction scheme given above, this can be explained by either (i) a fast singlet to triplet intersystem crossing or (ii) a fast internal conversion process from the $\mathrm{SW}^{*}$ state such as an intramolecular rearrangement into another nonemitting state. To discriminate between the various configurational possibilities of the $N$-phenyl derivative 3 , we have hindered the configurational changes of the molecule by increasing the viscosity of the medium; two different approaches have been used for this: first, by increasing the amount of glycerol in a glycerol/ethanol mixture at room temperature and, second, by decreasing the temperature of an ethanolic solution.

Viscosity Effects. The rotation of the phenyl group following the light absorption by molecule 3 has been demonstrated at room temperature by using several glycerol/ethanol mixtures. In ethanol, the main fluorescence occurs at long wavelengths, and it hardly decreases when the glycerol content is increased. However, a new broad and structureless emission appears (Figure 3) at the same time with a maximum at $405 \mathrm{~nm}$, and the breadth of this band is similar to that observed for the $\mathrm{N}-\mathrm{H}$ and $\mathrm{N}-\mathrm{CH}_{3}$ derivatives in this solvent mixture. The absence of structure for the $\mathrm{SW}^{*}$ emission can be attributed to the time taken for the glycerol/ethanol mixture to relax around the $\mathrm{SW}^{*}$ excited state. Cooling the $80 / 20$ glycerol/ethanol solution of 3 to $200 \mathrm{~K}$ makes the short-wavelength emission appear structured; this corroborates that the broad fluorescence band observed around $405 \mathrm{~nm}$ at room temperature is the superimposition of several $\mathrm{SW}^{*}$ emissions differing from each other because of the position of the surrounding glycerol molecules.

The rotational diffusion time $\tau_{\mathrm{r}}$ of the excited solute molecule in the solvation shell can be obtained from the Debye-StokesEinstein relationship

$$
\tau_{\mathrm{r}}=\frac{1}{k_{\mathrm{r}}}=\frac{\eta V}{k T}
$$

where $\eta$ is the viscosity coefficient of the solvent at the temperature $T, k$ is Boltzmann's constant $\left(1.38 \times 10^{-16} \mathrm{erg} \cdot \mathrm{K}^{-1}\right)$, and $V$ is the molecular volume of the excited molecule deduced from the van der Waals increments. ${ }^{29}$ The molecular volume calculated for $N$-phenyl-2,3-naphthalimide is ca. $202 \AA^{3}$ from which a relaxation time of $2.0 \mathrm{~ns}$ is calculated in the $50 / 50(\mathrm{v} / \mathrm{v})$ glycerol/ethanol mixture $(\eta=43.5 \mathrm{cP})$. This relaxation time clearly indicates that the molecule does not have the time to relax completely in the solvent mixture since the lifetime of the short-wavelength emitting state is ca. 4 times shorter than the solvent relaxation time.

However, the effect of viscosity on the fluorescence properties of 3 should not be attributed only to the variation in solvent relaxation time, since the branching ratio for the formation of the $\mathrm{LW}^{*}$ and $\mathrm{SW}^{*}$ states from the Franck-Condon state is likely to depend on viscosity which will influence the efficiencies of internal conversions from the excited states occurring by rotational (torsional) motion of the $N$-phenyl group. The $80 / 20(\mathrm{v} / \mathrm{v})$ glycer$\mathrm{ol} /$ ethanol mixture has a viscosity coefficient of only $220 \mathrm{cP}$ at

(29) Edward, J. T. J. Chem. Educ. 1970, 47, 261. room temperature; this value appears to be too small for changing considerably the $\mathrm{SW}^{*}$ and $\mathrm{LW}^{*}$ emissions ratio. For instance, under conditions where the viscosity coefficient is $600 \mathrm{cP}$, ethanol at $135 \mathrm{~K}$, the short-wavelength emission is still very weak (Figure 5).

It has been shown that increased solvent viscosity enhances both the fluorescence yield and the decay time of the SW" emission, thus indicating that a viscosity-sensitive deactivation process from $\mathrm{SW}^{*}$ operates. On the other hand, the fluorescence quantum yield and lifetime of $\mathrm{LW}^{*}$ are practically constant when the glycerol content of the glycerol/ethanol mixture is increased at room temperature.

The experimental ratios $\Phi_{f} / \tau$ may be used together with eqs 9 and 10 to obtain estimates for the yields of formation of the emitting states, i.e., for $\Phi_{\mathrm{SW}}{ }^{\prime}$ and $\Phi_{\mathrm{LW}} \mathrm{w}^{\prime \prime}$. Assuming $k_{f}^{\prime}$ for compound 3 to be the same as that for 1 and 2 (where $k_{f}^{\prime}=3 \times 10^{7}$ $\mathrm{s}^{-1}$ ), an average $\Phi_{f}^{\prime}$ yield of about 0.4 is obtained from the $\Phi_{f}^{\prime} / \tau$ ratio measured at room temperature. No good $k_{f}^{\prime \prime}$ estimate for 3 can be obtained; thus, a rough first approximation of $3 \times 10^{7}$ $\mathrm{s}^{-1}$ is assumed for it. With this value, a $\Phi_{L} w^{\prime \prime}$ yield of about 0.1 can be estimated; this value decreases as the solvent viscosity increases.

Although the experimental error of the $\Phi_{\mathrm{f}} / \tau$ ratios and especially of the $k_{f}^{\prime \prime}$ estimations introduces some uncertainties in the quantum yields given above, nevertheless the results indicate that $\Phi_{\mathrm{SW}^{\prime}}+\Phi_{\mathrm{LW}}{ }^{\prime \prime}<1$. This means that other processes compete with the formation of the $\mathrm{SW}^{*}$ and $\mathrm{LW}^{*}$ states from the FranckCondon state.

The lifetime of the short-wavelength emission of 3 increases as the viscosity of the glycerol/ethanol mixture increases while that of the long-wavelength emission remains practically unchanged ( $1.3 \mathrm{~ns})$. This observation can be taken as an indication that the deactivation route of $\mathrm{SW}^{*}$, but not of $\mathrm{LW}^{*}$, is hindered by increasing the viscosity.

Temperature Effect. The effect of temperature change on the luminescence properties is expected to arise from two effects. First, large decreases in temperature are likely to decrease the rate of activation-controlled processes, and, second, viscosity changes will induce the same effects as those observed for the ethanol/glycerol mixtures. There are no detailed data available on the temperature dependence of the lifetime of the short-wavelength emission. We know that in ethanol solution $\tau$ increases from the room temperature value of less than $50 \mathrm{ps}$ up to several nanoseconds at 100 $\mathrm{K}$. The processes depopulating the $\mathrm{SW}^{*}$ state might be intersystem crossing to the triplet state and internal conversion to the ground state through rotational or torsional motion of the $N$-phenyl group. Therefore, the considerably longer lifetime at $100 \mathrm{~K}$ is not unexpected at this low temperature where the molecule's motion becomes strongly inhibited.

The slight blue shift $(25 \mathrm{~nm})$ of the long-wavelength fluorescence as the temperature is decreased results from the increased solvent relaxation time around the excited molecule due to the higher viscosity. The rotational diffusion time $\tau_{\mathrm{r}}$ calculated from relation 14 gives $70 \mathrm{ps}$ for the ethanolic solution at room temperature $(\eta=1.2 \mathrm{cP})$. Such relaxations already have precedents. ${ }^{30-32}$ However, it becomes much longer, $35 \mathrm{~ns}$, when the temperature is decreased to $135 \mathrm{~K}(\eta \approx 600 \mathrm{cP})$. Then the solvent can be considered as motionless within the lifetime of the excited state emitting at long wavelength: $2.8 \mathrm{~ns}$.

The lifetime of the $\mathrm{LW}^{*}$ state of 3 also increases by a factor of 2.1 (from 1.3 to $2.8 \mathrm{~ns}$ ) when the temperature decreases from 293 to $135 \mathrm{~K}$ (Figure 6). In the absence of viscosity effects (vide supra), the smaller temperature dependence of the lifetime corresponds to the nonradiative processes determining the decay of the $\mathrm{LW}^{*}$ state.

The quantum yields of fluorescence emitted by $\mathrm{SW}^{*}$ and $\mathrm{LW}^{*}$ states of 3 increase with decreasing temperature in the range of

(30) Wintgens, V.; Pouliquen, J.; Valat, P.; Kossanyi, J.; Canonica, S. S.; Wilds, U. Chem. Phys. Lett. 1986, 123, 282.

(31) Lai, T.; Lim, B. T.; Lim, E. C. J. Am. Chem. Soc. 1982, 104, 7631.

(32) Arnold, K.; Yarwood, J.; Price, A. Mol. Phys. 1983, 48, 451. 


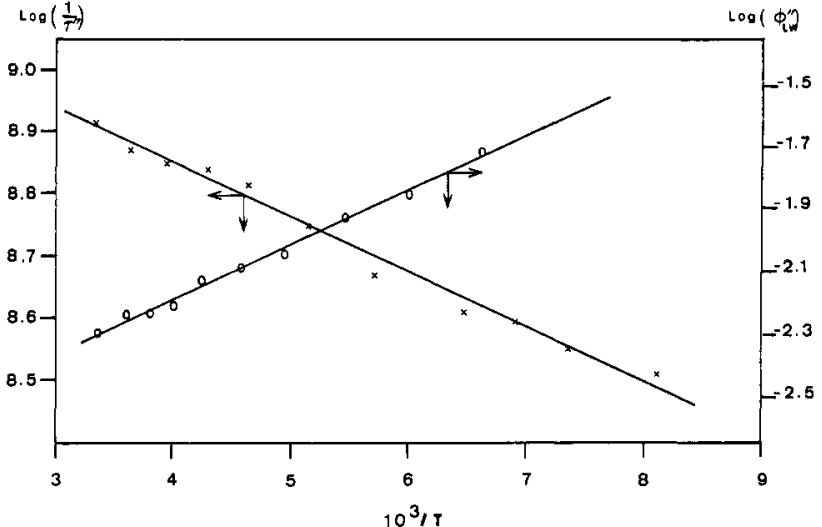

Figure 6. Variation of the logarithm of the long-wavelength fluorescence quantum yield $\left(\log \Phi_{L w^{\prime \prime}}\right)$ and rate constant $\left(\log \left(1 / \tau^{\prime \prime}\right)\right)$ of 3 versus $1 / T$ $\left(\mathbf{K}^{-1}\right)$ in ethanol solution.

$300-135 \mathrm{~K}$; this amounts to a factor of 7 for $\mathrm{LW}^{*}$. The fluorescence increase is small but real in case of $\mathbf{S W}^{*}$, but it would be hazardous to quantify it because of the very low efficiency in this temperature range.

Assuming that $k_{f}^{\prime \prime}$ is temperature independent, the ratio of LW* fluorescence quantum yield over its lifetime increases by ca. a factor of 3.2 between 298 and $135 \mathrm{~K}$, which, according to eq 10 , corresponds to an increase in the yield of formation of the $\mathrm{LW}^{*}$ state with decreasing temperature. Thus, the experimental temperature dependence of $\Phi_{L W}$ "is not in accord with what is expected. The high increased viscosity with decreasing temperature should not favor the formation of $\mathrm{LW}^{*}$, which needs rotation of the $N$-phenyl group to be formed. This discrepancy can be explained by assuming that the formation of $S W^{*}$ from the Franck-Condon state requires a small activation energy which determines the temperature dependence of the branching ratios for the formation of $\mathrm{SW}^{*}$ and $\mathrm{LW}^{*}$. For example, $\mathrm{SW}^{*}$ may be formed from the Franck-Condon state by intramolecular geometrical relaxation such as a change in the valence angles of the $\mathrm{N}$ atom.

A further decrease in temperature from about 135 to $77 \mathrm{~K}$ causes a sharp change in the fluorescence quantum yields: The $\mathrm{LW}^{*}$ emission becomes undetectable at $77 \mathrm{~K}$, while the $\mathrm{SW}^{*}$ emission increases to become the only strong fluorescence emitted at $77 \mathrm{~K}$. This characteristic change in luminescence behavior can be explained by the blocking of the $N$-phenyl rotation by freezing the solution. Since an energy barrier exists between the Franck-Condon and the SW* states, the structured emission observed at short wavelength below $110 \mathrm{~K}$ must originate from the Franck-Condon state.

Structure of the Ground and Excited States. It is important to confirm the structure of the molecules in their ground state, but the structures found in the literature determined by X-ray crystallography deal only with $\mathrm{N}$-substituted phthalimides. Furthermore, the results concerning the orientation of the $N$-phenyl substituent with regard to the phthalimide moiety are contradictory. Thus, dipole moment measurements suggest that $N$ phenylphthalimide has a preferred planar conformation..$^{33}$ On the other hand, X-ray crystallographic investigation of $N$ - $\left(2^{\prime}\right.$ carboxyphenyl)phthalimide indicates that the carbon atoms bound to the nitrogen atom are coplanar and that the $N$-phenyl and the phthalimide groups are in orthogonal planes. ${ }^{34}$ Similarly, X-ray diagrams of $N$-cyclohexylphthalimide show that the cyclohexyl ring is not coplanar with the phthalimide ring. ${ }^{35}$

An extensive X-ray analysis has been performed ${ }^{36}$ upon 3. It demonstrates that, in the solid state the two planes, the one

(33) (a) Lumbroso, H.; Dabbard, R. Bull. Soc. Chim. Fr. 1959, 749. (b) Ascoria, A.; Barassin, J.; Lumbroso, H. Bull. Soc. Chim. Fr. 1963, 2509

(34) Mayer, R. M.; Pratt, M. R. A. Acta Crystallogr. 1963, 16, 1086.

(35) Groth, P. Acta Chem. Scand. 1969, 23, 1076.

(36) We thank Dr. Y. Dromzee of the University Pierre et Marie Curie in Paris for having carried out this analysis.

containing the $N$-phenyl substituent and the other the 2,3naphthalimide part form a $60^{\circ}$ angle. Interestingly, the lengths of the two $\mathrm{N}-\mathrm{C}$ bonds between the nitrogen atom and the carbon atoms of the two carbonyl groups are almost identical (1.393 and $1.407 \AA$ ) but shorter than the $N$-phenyl bond $(1.425 \AA)$ or than the distance between the nitrogen atom and the carbonyl carbon atom $(1.426 \AA)$ in $N$-methylbenzamide, ${ }^{37}$ thus showing some double-bond character. In addition, the sum of the three angles made by the nitrogen atom and its three substituents is $359^{\circ} 50^{\prime}$, which clearly shows that the three carbon atoms bound to the nitrogen are coplanar with the latter.

MNDO calculations performed on $N$-phenyl-2,3naphthalimide ${ }^{38}$ indicate that the ground state of the molecule is stabilized by $\sim 0.5 \mathrm{eV}$ when the phenyl substituent is in a plane orthogonal to the naphthalimide moiety. In this case, the transition is of naphthalene-naphthalene type, in agreement with the fact that all four compounds 1-4 have almost identical long-wavelength absorption spectra.

No calculations could be carried out upon the excited state of 3 but only on that of $N$-phenylphthalimide. If the results obtained can be extended to the $N$-phenyl-2,3-naphthalimide series, then the excited state would have charge-transfer character involving a negative charge transferred from the phenyl group to the naphthalimide moiety. This is found to be stabilized by ca. $90^{\circ}$ rotation of the phenyl group in order to become coplanar with the naphthalimide part of the molecule.

Due to its slightly polar structure and according to the X-ray measurements, as well as to the deductions made from the spectroscopic data, the ground state of 3 and its Franck-Condon excited state in solution would have structure 5 , where the $N$ phenyl substituent is orthogonal to the naphthalimide moiety.

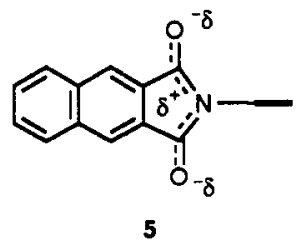

As far as the $\mathrm{LW}^{*}$ state is concerned, a structure in which the two aromatic moieties are coplanar enables an extended conjugation through the nitrogen lone-pair electron orbital. Such a structure would correspond to either one of the following two structures, 6 and 7, but according to the theoretical calculations

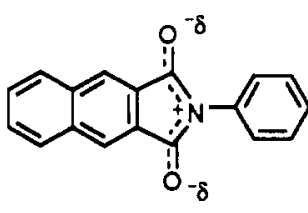

6

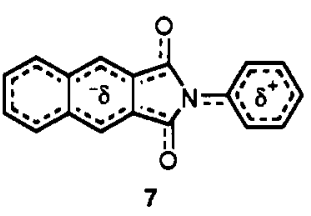

extended to the naphthalimide series (vide supra), the excited state would have some charge-transfer character coresponding better to 7 in which the charges are delocalized over the two coplanar aromatic moieties. Nevertheless, the low value obtained experimentally for the variation of the dipole moment between the ground state and the excited state $(\Delta \mu=3.4 \mathrm{D})$ is more in favor of the less polarized structure 6 which is similar to that of the ground state (with a planar nitrogen atom) with the exception of the rotated phenyl substituent.

There is already a precedent of dual emission for this type of chromophore with double-bond character between the nitrogen atom and the carbon atom of a carbonyl group. This occurs with benzanilide which emits only weakly from the Franck-Condon state but shows two other fluorescences, one of them originating

(37) Handbook of Chemistry and Physics, 61st ed.; CRC: Boca Raton, FL, 1980-81; p F-219.

(38) We thank Dr. P. Chaquin of the University Pierre et Marie Curie in Paris for letting us know the results of his theoretical calculations before publication. 


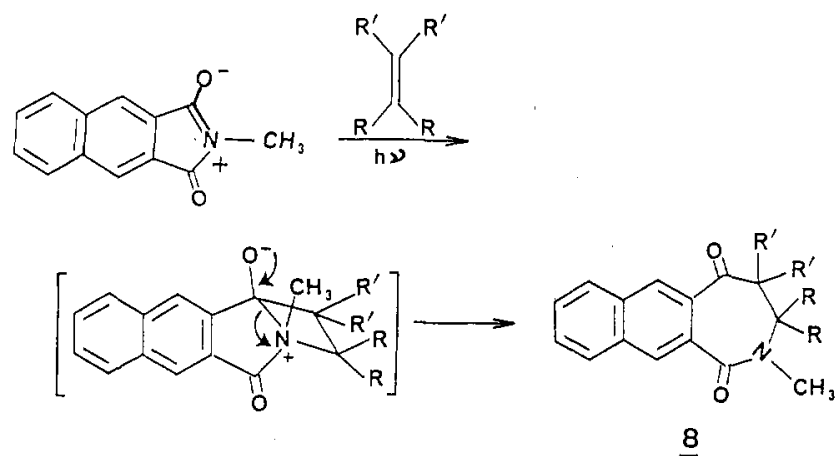

Figure 7. Reaction scheme of the photochemical addition of olefins to $N$-methyl-2,3-naphthalimide (2) leading to diazepinediones 8.

from an excited imidol structure in which the $\mathrm{C}=\mathrm{N}$ double bond has been created by double hydrogen transfer between two molecules. ${ }^{25,39}$

The creation of a double bond between the nitrogen atom and the carbonyl group of $N$-methyl-2,3-naphthalimide (2), which

(39) (a) Heldt, J.; Kasha, M. J. Mol. Liquids 1989, 41, 305. (b) Tang, G. Q.; Mac Innis, J. M.; Kasha, M. J. Am. Chem. Soc. 1987, 109, 2531. presents a rather long excited singlet lifetime (ca. $8 \mathrm{~ns}$ ), would explain its $2 \pi+2 \pi$ cycloaddition to an olefin 11 leading to the naphthazepinedione molecules 8 (Figure 7). Such an intermediate has also been proposed in the case of $N$-methylphthalimide ${ }^{14,15}$ and of $N$-methyl-1,2-naphthalimide; 40 it is closely related to the product resulting from the photoaddition of olefins to ethoxyisoindolone where the four-membered ring intermediate could be isolated. ${ }^{41}$

In summary, both the short-wavelength and the long-wavelength emitting states of 3 are found to originate from, but are not, the Franck-Condon state. They decay with lifetimes differing from each other by about 2 orders of magnitude. In both the Franck-Condon state and the short-wavelength emitting state, the phenyl substituent is in a plane orthogonal to the naphthalimide moiety, while in the long-wavelength emitting state the two planes are coplanar.

Registry No. 1, 6247-15-0; 2, 42896-23-1; 3, 21815-18-9; 4, 137769 13-2; 2,3-naphthalenedicarboxylic acid, 2169-87-1; 2,5-di-tert-butylaniline, 21860-03-7.

(40) Kubo, Y.; Toda, R.; Yamane, K.; Araki, T. Bull. Chem. Soc. Jpn. 1986, 59, 191 .

(41) Rodehorst, R. M.; Koch, T. H. J. Am. Chem. Soc. 1975, 97, 7298.

\title{
Existence of Carbanion Triplet Structures As Evidenced by NMR Spectroscopy and MNDO/AM1 Calculations
}

\author{
Ingmar Sethson, ${ }^{1 \mathrm{a}}$ Dan Johnels, ${ }^{1 \mathrm{a}}$ Tore Lejon, ${ }^{1 \mathrm{a}}$ Ulf Edlund, ${ }^{*, 1 \mathrm{a}}$ Brian Wind, ${ }^{1 \mathrm{~b}}$ \\ Andrzej Sygula, ${ }^{1 c, d}$ and Peter W. Rabideau*,id
}

Contribution from the Department of Organic Chemistry, Umea University, S-901 87 Umea, Sweden, Department of Chemistry, Indiana-Purdue University, Indianapolis, Indiana 46205, and Department of Chemistry, Louisiana State University, Baton Rouge, Louisiana 70803-1802. Received April 23, 1991. Revised Manuscript Received September 24, 1991

\begin{abstract}
Dilithium 2,2'-biindenide and 1,2'-biindenide have been taken as model systems to study the existence of carbanion triplet structures. Induced ${ }^{13} \mathrm{C}$ NMR chemical shifts by changing ether solvent indicated a polarization of charge toward the bridging positions as the cation solvation decreases. Moreover, in the unsymmetrical biindenide, a restricted rotation around the bridging bond could be manifested, governed by a negative entropy term. MNDO/AM1 calculations, where solvation and $\mathrm{C}-\mathrm{Li}$ interactions were accounted for, supported a carbanion triplet arrangement where the rotational barrier decreased by increased cation solvation.
\end{abstract}

\section{Introduction}

The nature of the carbanion-alkali metal interaction is an important aspect of carbanion chemistry. ${ }^{2}$ A variety of spectroscopic and theoretical approaches have been utilized to increase the understanding of this area of organometallic chemistry, where the role of covalency vs ionic interactions and also the presence of aggregation effects have been the greatest challenges. In the case of salts involving delocalized dicarbanions, an ion triplet arrangement has been proposed. ${ }^{3}$ This proposal was based on the application of Coulomb's law, where the alkali ions were

(1) (a) Umea University. (b) Indiana-Purdue University. (c) Louisiana State University. (d) Permanent address: Department of Chemistry, Jagiellonian University, Krakow, Poland.

(2) (a) Stowell, J. C. Carbanions in Organic Synthesis; Wiley: New York 1979. (b) Günther, H.; Moskau, D.; Bast, P.; Schmalz, D. Angew. Chem. $1987,99,1242$ and references therein.

(3) (a) Streitwieser, A., Jr.; Swanson, J. T. J. Am. Chem. Soc. 1983, 105,

2502. (b) Streitwieser, A., Jr. Acc. Chem. Res. 1984, I7, 353 treated as point charges. The model was used to explain the unexpectedly small difference between the first and second acidity constants in a number of hydrocarbons, especially $2,2^{\prime}$-biindene and 9,9'-bifluorene where $\Delta \mathrm{p} K_{\mathrm{a}}<1$. The ion triplet structure of dilithium $9,9^{\prime}$-bifluorenide has been confirmed in the solid state by X-ray crystallography. ${ }^{4}$ This simple model, and extensions of it, ${ }^{5}$ has further been used to account for the crystal structure of other dicarbanions. Of course, crystal packing or Madelung forces may be a complicating factor in the solid "lattices", but it is unclear to what extent such forces will favor or disfavor triplet structures in a given complex.

Some semiempirical MNDO calculations have also been performed in order to investigate the structures of dilithium dicarbanions. ${ }^{5,6}$ In one MNDO study, ${ }^{6 \mathrm{~d}}$ the ion triplet structure was

(4) Walezak, M.; Stucky, G. D. J. Organomet. Chem. 1975, 97, 313.

(5) Grovenstein, E., Jr. In Comprehensive Carbanion Chemistry, Part C; Buncel, E., Durst, T., Eds.; Elsevier: Amsterdam 1987; p 175. 